\title{
Optimal Planning for Cellular Networks for Smart Metering Infrastructure in Rural and Remote Areas*
}

\section{Óptima Planeación de Redes Celulares para la Infraestructura de Medición Inteligente en Zonas Rurales y Remotas}

DOI: http://dx.doi.org/10.17981/ingecuc.11.2.2015.05

Research Article - Reception Date: February 27, 2015 - Acceptance Date: September 15, 2015

Paúl Masache

Electrical Engineer, Universidad Politécnica Salesiana. Quito. (Ecuador).pmasache@ups.edu.ec

Esteban Inga

Master in Education and Social Development, Universidad Politécnica Salesiana. Quito. (Ecuador). einga@ups.edu.ec

\section{Roberto Hincapié}

Doctor in Engineering, Universidad Pontificia Bolivariana. Medellín. (Colombia). roberto.hincapie@upb.edu.co

To reference this paper:

P. Masache, E. Inga and R. Hincapié, "Optimal Planning of Cellular Networks for Smart Metering Infrastructure in rural and remote areas," INGE CUC, vol. 11, no. 2, pp. 49-58, 2015. DOI: http://dx.doi.org/10.17981/ingecuc.11.2.2015.05

\begin{abstract}
Smart metering is used to control, monitor and know the system status in real time; to this effect, the incorporation of smart grids primarily benefits the electrical system; similarly, the reuse of infrastructure and cellular spectrum help mitigate the time and cost of its implementation. In order to reduce traffic and saturation of cellular networks, this paper aims at determining the optimal route for information transmission analyzing the optimal routing through distances and optimal routing through traffic flow. This analysis helps determine what the optimal route is, when there is no traffic on the wireless network, or when there is prolonged traffic, and what the traffic tendencies are, that may occur by excessive information transmission of smart meters to electric distribution companies.
\end{abstract}

Keywords - Smart Grid, Smart Metering, AMI, Cellular Network, Optimal Planning, Traffic, Optimal Routing.
Resumen- La medición inteligente se emplea para controlar, monitorear y conocer el estado del sistema en tiempo real; por ese motivo, la incorporación de redes inteligentes beneficia primordialmente al sistema eléctrico. Así mismo, con la reutilización de la infraestructura y del espectro celular, ayuda a mitigar el tiempo y el costo de su implementación. Con la finalidad de reducir el tráfico y la saturación de las redes celulares, se propone determinar la ruta óptima para el envío de la información, para ello se analiza un ruteo óptimo por medio de distancias y un ruteo óptimo por medio de flujo de tráfico. Gracias a este análisis, se determina cuál es la ruta óptima cuando no existe tráfico en la red celular o cuando existe un tráfico prolongado, y cuáles son las tendencias de tráfico que se pueden producir por el envío excesivo de la información de los medidores inteligentes hacia las empresas eléctricas de distribución.

Palabras claves - Red Eléctrica Inteligente, Medición Inteligente, AMI, Red Celular, Óptima Planeación, Tráfico, Enrutamiento Óptimo.

\footnotetext{
* Research article derived from research project entitled "Modelamiento y simulación de redes inalámbricas para la medición inteligente de energía eléctrica residencial rural”. Funded by the Universidad Politécnica Salesiana y Grupo de investigación en redes eléctricas inteligentes GIREI. Starting Date: February 06, 2014. Compleition Date: March 20, 2015.
} 


\section{INTRODUCTION}

With the increase of electricity consumption and the creation of new sources to meet electricity demand, it is essential to have a system that is able to improve performance and maximize energy sources; for that reason, a smart system helps to improve the network. Smart Grid is a concept that encompasses a number of benefits. A Smart Grid helps improve the distribution system and also helps with the planning of the system by monitoring and, controlling it which means that the implementation of a Smart Grid in the distribution system enables greater efficiency in respect to a traditional system [1]. The traditional system did not have a large number of interconnections, its demand was minimal and its most widely used topology was radial as it is easier to control. However, with the improvement of infrastructure, a closed topology, better control and monitoring was needed to ensure service [2]. Some benefits of the implementation of a Smart Grid worth mentioning are, on the one hand, there is a better distribution of electrical energy in a system which is controlled and monitored in near-real time with high-speed transmission, that is, it implies the existence of an advanced metering infrastructure (AMI) [1] [3]. On the other hand, another key aspect of the implementation of a Smart Grid is to have the ability to provide security to the system [4] against external agents that may be introduced in it in order to destabilize it or hackers who wish to have classified procedure information, control of resources and services provided to different consumers [5] [6].
In order to have a conformed Smart Grid, an infrastructure to monitor and control should exist, that is, an AMI system, as it improves the efficiency of the distribution network, optimizes operations and controls system losses [7] [8]. One advantage for consumers is the ability to determine when the best moment to consume the highest amount of energy is, as it is possible to provide in real-time what their energy consumption is, in that instant of time, and thus the users can enhance their consumption and distribution companies can control the distribution of energy [9] [10] [11]. In order to achieve the latter, the AMI network should operate at high speed to provide excellent service and allow the consumers to have a greater share of energy consumption because with consumption display, they can optimize and enhance their demand occurring in the peak hours [12] [13]; for this purpose, AMI is specified in three key points of operation: first, in measurement of demand and equipment control found in a distribution system, as this will help to have a better scope of action when a fault occurs in the system and thus reduce network losses [14]; second, in software that has the ability to control the system and can display in real time each of the sections of the network, and thus, monitor the system in each instant of time; it can be done with the implementation of SCADA systems or Remote Terminal Units (RTU) for obtaining signals [15]; and finally, in an adequate communication infrastructure, as it is essential to have communication between the network and the operation equipment and maintenance. Here it is noteworthy that in relation to infrastructure

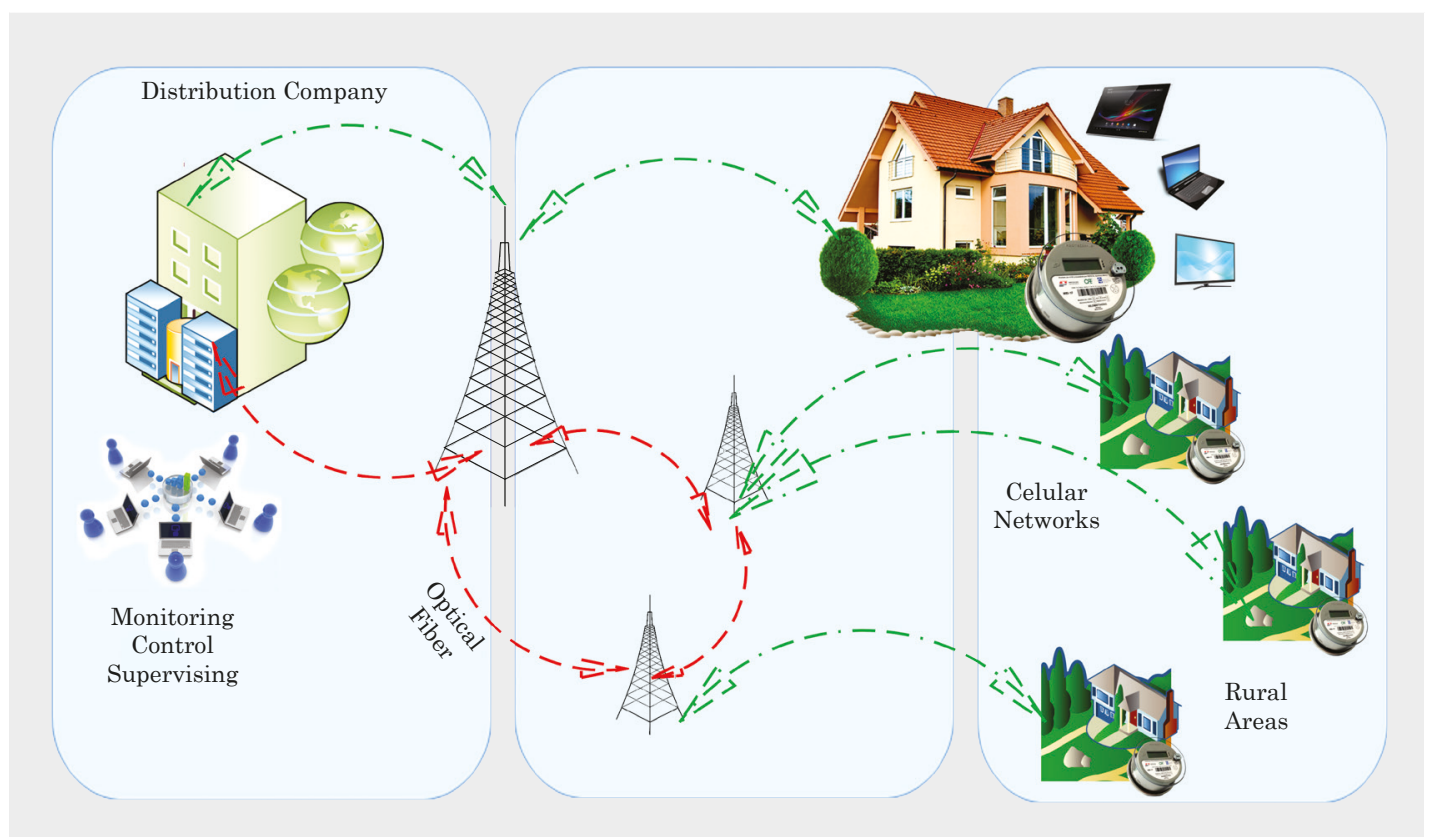

Fig. 1. Smart metering infrastructure. Source: Author 
optimization and cost reduction, an infrastructure that is already in use, can be reused such as a cellular network GPRS, CDMA or LTE [16] type [17]; for example, in cities there are a variety of networks that promote enhanced communication, such as a GPRS cellular network or a fiber optic network, but instead, if in rural areas, the landscapes changes, as it is away from the center of trade, reuse of equipment is lower. If this is the case, it is possible to reuse a CDMA cellular network to areas where there is coverage and a cognitive TV network [18] where the coverage of cellular networks is minimal.

With the implementation of smart metering, as AMI, many factors in the systems are improved, for example, issues such as the collection of information sent from the target consumers. This form of obtaining information makes the system easier to monitor, but it is required an implementation of an improved modern infrastructure which can census measurement devices. A clear example is shown in Fig. 1, a cellular network for station communication with smart meters and optic fiber network for communication between base stations and electric distribution companies is used; however due to the large investment that has to be done for its implementation, it is necessary the reusing of an existing infrastructure. This infrastructure serves communication between measuring devices and servers that will store data, also for the companies that are responsible for controlling the system and the information provided by the devices. [19] [10].

AMI provides the user with knowledge of their consumption, and thus, the user chooses to maintain consumption and pay its bill according to what they are consuming, or optimize their consumption, that is, only consume what is necessary and thus improve billing. AMI not only helps users, but also companies that supply energy permitting the knowledge of the losses that exist in the system; when a fault occurs, it is controlled and service is restored in a minimum time. In short, the AMI infrastructure makes the system more controllable in real time. All this leads to determine that to have full control of the system, it is required the implementation of an AMI [20] [11] [21]; yet to implement an AMI system, important aspects need to be considered: the system should be monitored for status and operation at all times; there should be full control of each of the measuring equipment, primarily in the sending of data from the equipment to monitoring and energy distribution companies.

For optimal monitoring and adequate control, there should exist an infrastructure that connects control centers with measuring devices; and lastly, good and safe handling of information [14] [22].

The problem that exists is the implementation of the necessary infrastructure to perform all of the above, since the investment in new infrastructure for communication between control companies and mea- suring equipment is very high because base stations in each sector and several concentrators that collect signals from the equipment and send its information to the base stations are to be built. Thus it is advocated the use of an infrastructure that is already in operation and which does not have to incorporate new base stations, but which allows the reusing of existing infrastructure to provide other services such as a cellular network. This network can be used for equipment communication, to do so, the spectrum released from primary consumers (mobile) is reused, since the measuring equipment come as secondary consumers to avoid traffic that can be produced by saturating the communication channels. In short, the main problem is to avoid the saturation of networks that are intended to be reused; therefore, a traffic analysis is carried out through the incorporation of smart meters in cellular networks in order to mitigate or reduce traffic through various optimization algorithms, such as optimal distances algorithm and optimal routing of traffic flows. [23] [24].

To use a cellular network is necessary to avoid saturating the channel, that is, to try not to congest the network that is used by the primary operators. Therefore, it is important to use a mobile virtual network operator (MVNO) [25] [26], because this operator informs about the channels that are available and if these channels can be reused for the sending and receiving data, primarily the transmission of information [27] [28].

Henceforth, this article is organized as follows: sections II, reusable cellular networks in rural areas are detailed; in Section III, algorithms for optimal planning of cellular networks are used; and finally, in section IV the respective conclusions are established.

\section{AMI Cellular Networks}

A wireless network is a network that has the greatest coverage and propagation. It is a network that has a wide variety of technologies and can be used in different areas, whether urban, sub-urban or rural; for that reason, it is determined the feasibility of reutilizing it to incorporate smart metering.

\section{A. LTE}

LTE stands for Long Term Evolution, which is fourth-generation technology. It is a migration of third generation technologies, and as such, it has more flexibility to send data and a bandwidth of about 180 $\mathrm{KHz}$. As a result, it provides great improvement in the transmission quality and the service using the network spectrum. One of the advantages of using LTE is the ease of passing data to different second or third generation technologies. The main objective of LTE is to lower latency among connections and to increase the transmission speed of data [29] [30]. 
The LTE standard works on the E-UTRAN and UTRAN architectures. E-UTRAN is a topology where connections can occur between base stations and controllers. The great advantage of this architecture is that there is communication between controllers. However, in a UTRAN [30] [31] architecture the connecting shape is different; the shape of the topology is a star where there is only connection among base stations but non-existent among controllers [32]. Apart from this, LTE uses the PHY physical layer with duration frames of no longer than $10 \mathrm{~ms}$. Each frame consists of 20 parts or periods with duration of $0.5 \mathrm{~ms}$. Additionally, LTE uses two types of technologies for downlink and uplink [33]:

\section{OFDMA (Orthogonal Frequency-Division Multiple Access )}

This division is done depending on the number of sub-bands that are needed and each sub-band has a transmission speed; this speed depends on the services to be allotted under the sub-band. Each subband makes an information code to avoid errors in the information. OFDMA is technology similar to CDMA; OFDMA's great advantage is the reduction of interferences in the connections and the increase in the transmission rate [32]. This technology is based on CSMA for multiplexing the information and uses the PHY physical layer. LTE uses OFDMA for information (downlink); to do so, it works within the operating bands that range from 1.25 to $20 \mathrm{MHz}$, and can hold 12 carriers with a transfer time of $0.5 \mathrm{~ms}$ per carrier [29].

\section{SC-FDMA (Single Carrier Frequency Division Multiple Access)}

A signal sent by SC-FDMA can be worked both in time domain and frequency domain; the way SCFDMA is transmitted is almost similar to OFDMA transmission, the big difference is that SC-FDMA has only one carrier [29]. SC-FDMA is used to send the information, i.e., to uplink since OFDMA has many disadvantages to send the information by having several carriers. This technology has the same band characteristics as OFDMA; it has the same band range from 1.25 to $20 \mathrm{MHz}$ with a total of 12 sub-carriers with a frequency of $15 \mathrm{KHz}$ per sub-carrier; the big difference between OFDMA and SC-FDMA is how the sub-carriers are integrated. OFDMA has a central subcarrier which is not used for mitigating interference, however, the SC-FDMA is a set of continuous subcarriers, i.e., there is no central subcarrier and for mitigating interference, it uses a pre-coding based on DFT, and thus the interference is dispersed throughout the bandwidth, decreasing in this way the interference damage [32].
Distortions in the paths of information can be present in both OFDMA and SC-FDMA. Apart from this, LTE uses OFDMA for the downlink of information, while SC-FDMA is used to send information (uplink). LTE has the same characteristics as Wimax in its operation; the big difference is the approach that each one has: LTE is used for services in urban areas and Wimax is highly used for services in rural areas.

\section{B. CDMA}

The acronym comes from Code Division Multiple Access; this technology was implemented in the second generation of cellular networks and uses multiplexing methods and codes for transmitting information, and once encoded, it uses the expanded method for transmission from the sender to the receiver of the data. As mentioned above, CDMA comes from the second generation CDMA ONE, which is adapted and improved in the third generation, CDMA2000 and CDMA450. CDMA requires access codes to link communication. These codes are provided to users automatically, and the information is encoded according to the code issued for the link. With this, security is improved because only code carriers may obtain the information details. The transmission speed, its coverage area and the propagation distance will depend on the type of technology that is being implemented; technology that stands out is CDMA450 and CDMA2000. Furthermore, CDMA can be combined with other technologies to improve their service, such as satellite communication that works under the frequency [34] that ranges from 11.7 to $14.5 \mathrm{GHz}$; this technology is very sensitive to climate change and interference and for that reason, it is combined with CDMA technology to improve data transmission and mitigate interference. The big disadvantage is that its implementation is more expensive, given that fact that two technologies are being implemented to improve service [35].

For communication, CDMA provides access codes between partners; these are called PN codes. Each user has a different code for transmission containing the information sent, thus the receiver can only access the code sent to that user, while the other codes are detected as noise. The coding to be sent is done through orthogonal functions, for this reason, the receiver can only access the information sent to that user. One advantage of CDMA technology is its hybrid domain, as it works in both time domain and frequency; it is a combination of TDMA and FDMA technologies [36]. TDMA technology refers to an assignment of the channels by time slots; it is more commonly used by the satellite and GSM technology; to avoid interference between the allocated zones there is a guard period. On the 
other hand, FDMA technology is a frequency allocation, i.e., it divides its bandwidth into frequency frames for each of the users who have coverage. The most common applications for this technology are the satellite repeaters and those communications which are still transmitted in analog form. As in TDMA, FDMA has a guard band to avoid interference between divisions [35].

This article shows the technologies that are feasible for spectrum reuse and for providing smart metering, which are suitable in rural and remote areas for their propagation and base station coverage. There are projects in which communication networks such as GSM, GPRS, via satellite and PLC are used to send data from substations and transformers to the central communication system. The mentioned project has improved the quality of service provided to customers, including a direct management of smart meters.

\section{III.MethodolOGY}

The methodological analysis was divided into two main parts: an analysis of optimal routing through distances and an analysis of optimal routing including the traffic that may occur in the cellular network for the integration of smart meters in the network. It is noteworthy that the analyses were performed opting for smart meters that work as secondary operators in the network.

\section{A. Optimal Routing Through Distance}

Each of the algorithms fulfills a vital function for the analysis of optimal routing. The employed analysis works in places where an improvement in handling of the electrical system is needed, and primarily, where a greater stability at the energy supply to customers is required. The pattern formed by these two algorithms is described below.

For proper operation of the analysis method, the different data or variables that are to be introduced in the algorithms should be established. These data are:

Base stations (BS); electrical distribution companies (EC); the values for the number of electrical distribution companies (Nurs); minimum and maximum number of users per cluster (MaxUsr, MinUsr); maximum distance allowed for optimum connection (dmax); number of base stations that will link companies $(\mathrm{K})$; from which place the information is sent (src); the place this information is bound (dest); importation of the geographic coordinates (GC) from the different distribution companies (longitude, latitude); and the weight assigned to the coordinates (dweight).

Necessary calculations are performed and in (1) the problem statement is specified to minimize the use of cellular networks in order to reduce transaction by smart meters. Calculation of each of the feasible links through the Euclidean distance is performed; for geo-referenced points, the Haversine formula should be used:

$$
d i s t=\sqrt{ }(X i-X o)^{2}+(Y i-Y o)^{2}
$$

In (2) a comparison is made between the calculated distances and the minimum allowed distances, as minimum distance link between existing or feasible nodes.

$$
\text { dist } \leq \text { minimum dist }
$$

Kmeans algorithm is used to group distribution companies $(E C)$ to each one of the base stations $(B S)$; a comparison with the various restrictions is performed; base stations and distribution companies are located on the map. The data required by the algorithm for its functioning are $K$ and the geographical coordinates of the distribution companies (GC): longitude and latitude. These restrictions can be seen in (3) and (4):

$$
\begin{aligned}
& \text { Nurs }>\text { MaxUsr } \\
& \text { Nurs_min }<=(\text { MinUsr - 1) }
\end{aligned}
$$

Where the number of users and the maximum number of users per cluster are compared. The Kmeans algorithm model is presented in (5):

$$
[i d x, \operatorname{ctrs}]=\operatorname{kmeans}(X, K)
$$

Where: idx is the link from base stations (BS) to each geographical coordinate (GC); ctrs are the optimal base stations (BS) determined by Kmeans. The $X$ and $K$ are the input variables of the algorithm, and instead ctrs and $i d x$ are outputs.

The distance between each of the geographical coordinates of the distribution companies (EC) and the base stations (BS) is calculated, distances are compared to the maximum connection distance; and the relevant restrictions to further optimize the mathematical model are performed. The mentioned restrictions are (6):

$$
\operatorname{dist}(i, i)=i n f
$$

This restriction places "equal to infinity" on the main diagonal of the matrix in order to avoid getting the optimal route in the same GC, as shown in (7).

$$
\operatorname{dist}(\operatorname{dist}>\operatorname{dmax})=\inf
$$


This restriction modifies distances greater than the allowable link distance, as shown in (8):

$$
\operatorname{dist}(1: \text { end }, m l+1: \text { end })=i n f
$$

These three restrictions belong to the geographical coordinates of the distribution companies (EC). Where dist refers to the distance between (EC), as shown in (9):

$$
\operatorname{distp}(i, i)=\inf
$$

The concept of restriction is the same as above. First, the main diagonal becomes infinite as shown in (10):

$$
\text { distp }=(\text { distp } / \text { dmax })>\text { dweight }
$$

The distance, which should not be greater than the set weight, is evaluated in order to eliminate saturated routes as shown in (11).

$$
\operatorname{distp}\left(\left(\operatorname{distp}{ }^{*} d \max \right)>\operatorname{dmax}\right)=i n f
$$

In (12) the permitted greater distance is re-evaluated, the big difference is the addition of dweight in the above restrictions.

$$
\operatorname{distp}(1: \text { end }, m l+1: \text { end })=\inf
$$

Instead the restrictions listed above represent the geographical coordinates (GC) of the base stations (BS); where distp is the distances between BS.

After obtaining the distances, the next step includes the use of Dijkstra's algorithm to find the optimum route to send the data, starting from one of the candidate sites and getting to another of the candidate sites, that is, one of the EC, as shown in (13):

dijkstra(ml, distp, origen, destination, 1:ml, 1:ml) (13)

Where Dijkstra represents the name of the function containing Dijkstra's algorithm; and $m l$ represents the number of BS that yields Kmeans algorithm.

\section{B. Optimal Routing Through Traffic Flow}

Dijkstra's algorithm is frequently used to find the optimal route through the connection distance between base stations or between the smart meters. For the next modeling, traffic flow is performed in order to determine an optimal route according to the link capacity and the saturation level that is found at any given time.
Traffic flow helps to determine congestion that is generated by sending information on a route on which there is saturation by excessive use of the same communication path. This traffic flow will help determine the saturation capacity of a network and what the favorable delivery route will be. In short, traffic flow summarizes operations to meet optimal routing but taking into account the traffic produced by using communication networks.

Lp-solve program is a very useful tool for optimization. This program calculates the necessary restrictions in order to find the best result for the end function; it offers tools both for minimizing and maximizing the entered resources. Data from Dijkstra's algorithm is introduced in order to find an optimal routing in accordance with the restrictions placed on files that were created from the program lp-solve; therefore, Matlab takes over each of the files created in the lp-solve program(14) and (15).

Before the entry of the variables, "mst.lp" and "routing_cap.lp" files, which were created in the program lp-solve, are named. Each of them is responsible for the restrictions and possible connections that can exist on different nodes. To this end, the file named "mst.lp" is responsible for the interconnections of the distribution companies (EC) to the base stations (BS), while the "routing_cap.lp" file is responsible for the possible connections between existing nodes.

$$
\begin{aligned}
& \text { fopen('mst. lp', 'w') } \\
& \text { fopen('routing_cap.lp','w') }
\end{aligned}
$$

The variables are entered and each are required for the analysis: $\mathrm{N}$, the number of stations; dmin, minimum connection distance; $x$ and the variable $y$, which are random dots that program finds as best connections; dist, the connection distance between BS and also, each of the feasible connections between nodes. Every traffic connections, traf, is calculated and the availability of each of the available connections, as well as their occupancy rate, busy connections.

Restrictions that were created after the execution of the file "mst.lp" in Matlab are obtained. The restrictions may be issued as follows:

- Restriction on the connection of active links

- Restriction on the number of existing links.

- Optimization of the program after assessing the restrictions.

These parameters are important to calculate feasible connections between different BS. These connections will be evaluated by the restrictions and the $d$ min set as an input variable, as shown in (16):

$$
\text { dist }<\text { dmin }
$$


After running mst.lp program, assessing the possible restrictions, and finding the feasible link, Dijkstra's algorithm is used to find the optimal route of the base stations (BS). To run the algorithm, we use the following input values, where src is the starting point of connection and dest is the end point of the connection, as shown in (17):

$$
\operatorname{dijkstra}(N, \text { dist, src, dest, } 1: N, 1: N)
$$

To perform the traffic analysis, the level of availability that available connections have, is used; this availability provides the network capacity that is not utilized, where the information can still be sent. The sent data, sentdata helps to determine the value of what is intended to send and it also helps to check for available transmission capacity through that connection, as indicated in (18):

$$
\text { available - sentdata }<\text { sentdata }
$$

Once the optimization of the feasible route is done through the availability matrix of a traffic flow, analysis is performed as follows:

The second analysis is performed with two operation charts, where the data to be sent and received in the feasible connections between BS are determined. The linking capacity of each of the BS and the information sending and reception tendency can be appreciated. To calculate the tendency of each of the nodes, it was applied (19):

$$
A R D=\frac{\text { average received data(in) }}{\text { average sent data (out) }}
$$

ARD is a formulation that helps to determine the average value of the received data and the sent data in each of the nodes.

To do this, the lp-solve program file called "routing_cap.lp" is used; this file is responsible for sending the connections between nodes and the data being sent and received from each BS. The routing restrictions of each $\mathrm{BS}$ and the feasibility of connecting each BS are applied in this program. Based on these restrictions, sent and received data are calculated. Also, to determine the ARD value, an analysis of the queuing system is performed which helps to determine what is the percentage of all the data transfers (received and sent) that was successfully completed; and the percentage that did not reach a successful transmission, that is, those data in which communication was not feasible. For this, the analysis of queues using the exponential distribution is used, as shown in (20):

$$
P=\lambda e^{\left(-\lambda^{*} A R D\right)}
$$

The uplink and downlink data help to calculate the communication average capacity, the uplink average capacity and downlink average capacity.

For a better understanding of the employed methodology, the following is the pseudo-code that is used in order to have a clearer picture of each of the steps required for the analysis of optimal routing through traffic flow.

\section{Pseudocode of optimal routing through traffic flow}

Step 1: $\mathrm{N}=300, \mathrm{Dmin}=0.3$;

Step 2: fopen ('mst.lp', 'w'); restrictions fopen

('routing_cap.lp', 'w');

Step 3: While Flag == 0; Calculation of $\mathrm{x}, \mathrm{y}$,

dist (random values).

Step 4: target function, constraints

Step 5: Dijkstra (src, dest, dist); optimal route

Step 6: available, busy, traf

Step 7: While t $\sim$ = source; Available - sentdata $<$ sentdata

Feasible route

Step 8: (in) / (out); ARD function

Step 9: $\lambda \mathrm{e}^{\wedge}\left(-\lambda^{*} \mathrm{ARD}\right)$; queuing system

\section{RESULTS}

For the presentation of the two simulations, taking into account an optimal distance and traffic analysis in the communication lines, and a better understanding and the pondering of feasible and optimal routings, random links were used with restrictions by a minimum connection.

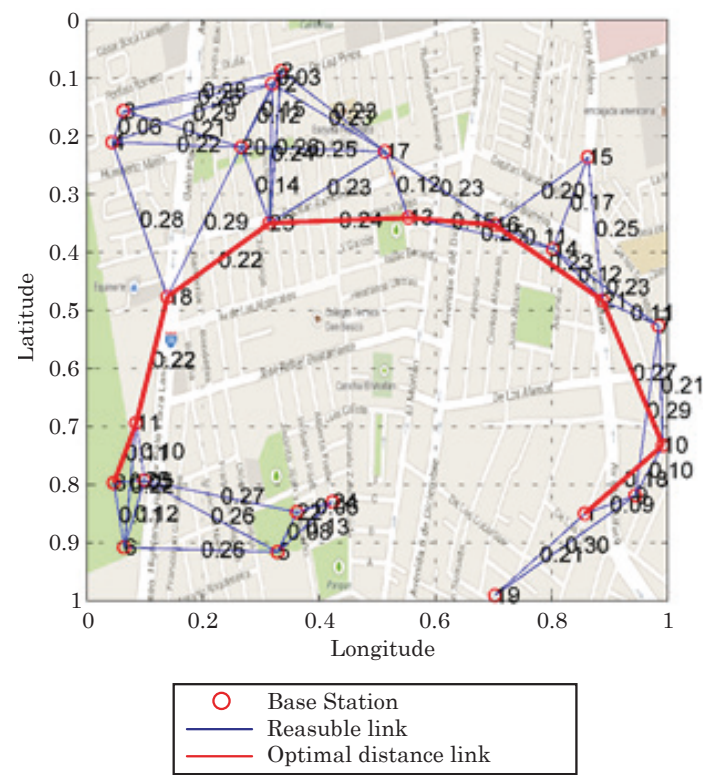

Fig. 2. Optimal routing for distances. Source: Author 
Feasible connections are those where you can send the information. These connections may be available for sending data, this will depend on how congested the network or its level of use at any given time. This process has already been demonstrated in the above analysis, however, it only relates to the shortest distance for sending the information required by the distribution companies; it does not assess the level of traffic that may be on each of the connection routes. It is a very unlikely outcome since the route shown in Fig. 2 or Fig. 3, may be the optimal distance, the shortest, but not the most feasible, since different situations indicate that the route should be modified in order to be able to send information without the need to wait for the transmission.

It is also observable that a routing through distances will be the most congested route, since this route is where the information is to be sent because sending time is reduced and shorter frames are equivalent to a minimization of the use of the spectrum or bandwidth that is intended to be used. It is also essential to know the size of the information to be sent and the capacity of each of the links with the finality to have an adequate simulation of each of the resources that are to be detailed. For these reasons, distance routing is feasible in case of adding a new architecture which is intended to optimize resources for the implementation of this architecture.

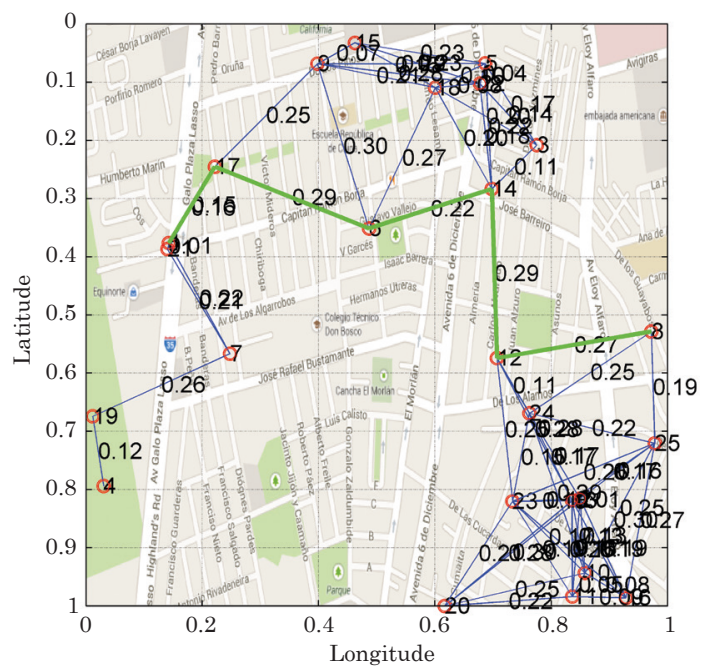

\begin{tabular}{|ll|}
\hline O & Base Station \\
\hline & Reasuble link \\
& Optimal distance link \\
& Optimal traffic link \\
\hline
\end{tabular}

Fig. 3. Optimal routing considering network traffic. Source: Author

Traffic is one of the factors that influence optimal transmission, and further, when trying

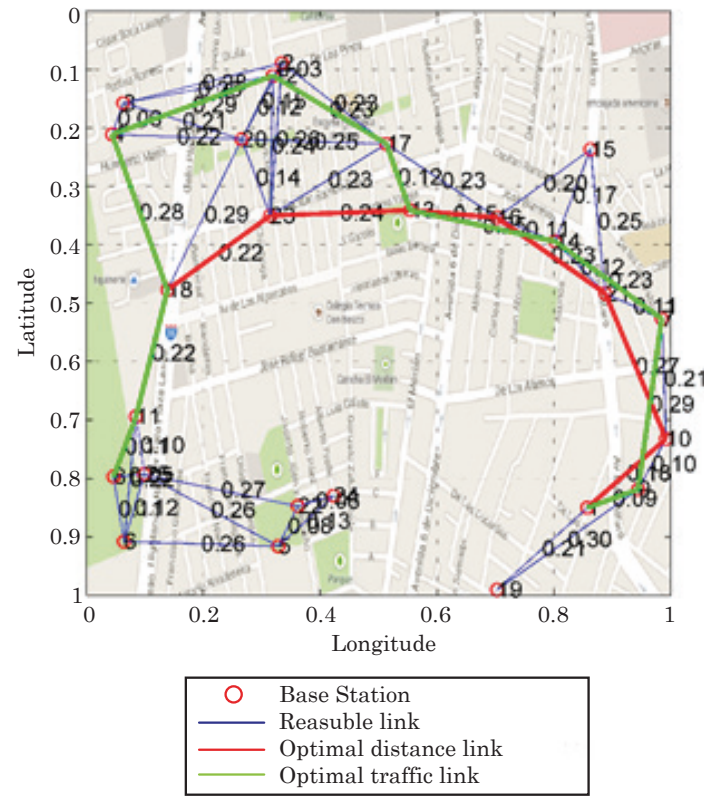

Fig. 4. Optimal Routing of distances and traffic. Source: Author

to reuse an infrastructure which already has a saturation range; therefore, traffic analysis in transmission networks for implementing smart metering as a service to the distribution system is critical. Through Fig. 2, Fig. 3 and Fig. 4 it can be shown the exposed difference, where it is shown that a distance route is not always the optimal path for sending of information, since traffic from one communication line can make changes in the original design; as a result, a big difference between the two optimal routes can be observed. Perhaps, the green marked route is not the most optimal route for the time of use of the infrastructure and the distance used for the sending, but it's the feasible route, the available route that exists at a particular time. However, for reasons of reliability, to prevent bandwidth saturation from occurring, and also depending on the level of importance of the information, it is the best route for sending. For a better understanding of the above, see Fig. 5.

According to the above it is demonstrated the importance of knowing the traffic of a system, still Fig. 5 and Fig. 6 show how the transmission of data is, both the sent data and the received data. It may be mentioned that an increase in the number of clients that send information produces the existence of an information range that is not possible to transmit since it creates communication traffic, therefore saturation in communication begins. The purpose is to determine how to avoid communication traffic and saturation of the transmission medium. 


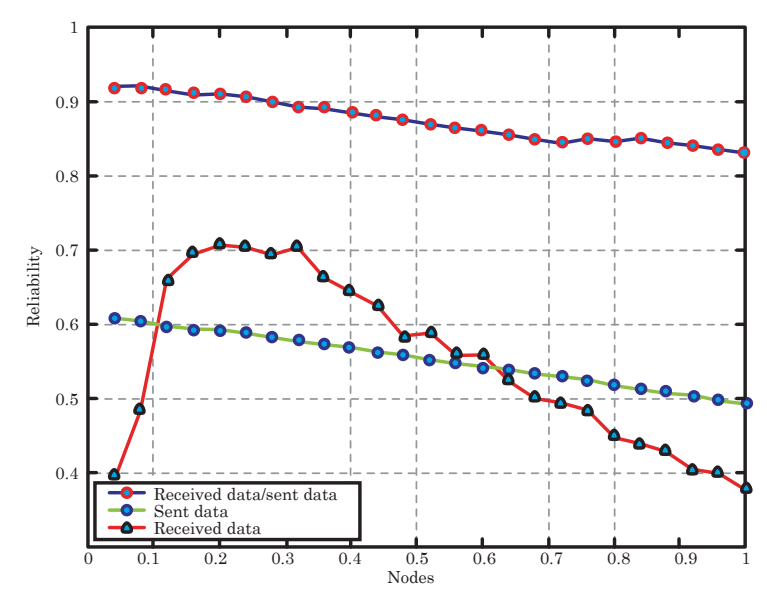

Fig. 5. Tendency analysis according to traffic routing. Source: Author

Fig. 6 shows the tendency of the information that cannot be sent or is lost when all information is transmitted at the same time; this information causes network traffic and for this reason, it is essential to analyze and study the network congestion in order to improve and expand both infrastructure and technology used for communication of primary and secondary operators. For this expansion or growth of communication infrastructure the range of traffic being produced and the coverage of each of the base stations must be known. One of the determinants of growth is the increase in customers; however, the annual percentage increase in customers and the ability of the existing infrastructure must have a match to reduce data loss, either by saturation of the means of communication or by the traffic being produced. This analysis involves all types of networks, as the network flow determines the expansion and an optimal infrastructure provides a better service; traffic determines the behavior of the transmission medium of the resources.

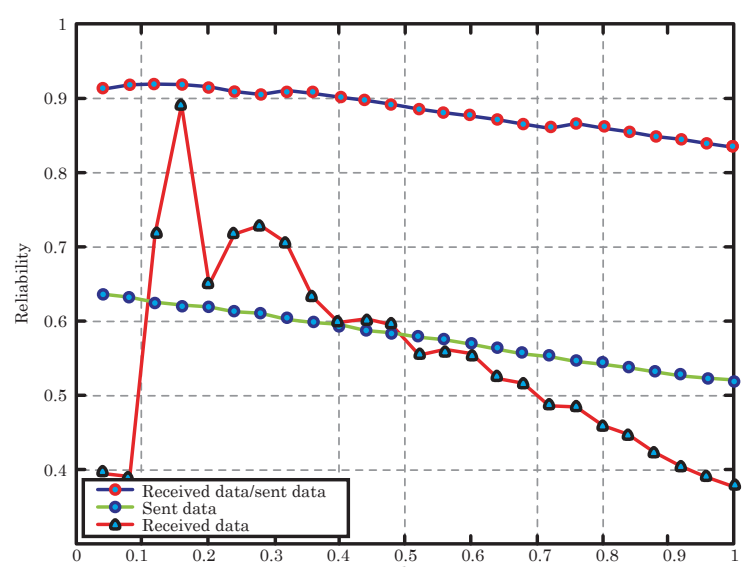

Fig. 6. Tendency graph by traffic analysis. Source: Author
Fig. 5 and Fig. 6 show how traffic from smart meters can affect communication in both sending and receiving data. For that reason, it is essential that smart meters work as secondary operators; since the produced traffic can cause a breakdown in communication and the integration of smart metering as a service to the electrical system. One of the feasible points is to determine the precise moment to send the information. Fig. 6 shows how improper use of the network would deploy saturation and high communication traffic.

\section{Conclusions}

Notably, the routings presented in this article help to minimize the network traffic and avoid congestion on cellular networks. These routings benefit the sending and receiving of data, and the accuracy of the sent information since the electrical system information in real time would be available.

Reusing existing infrastructure helps us to implement in less time the metering service and it optimally reduces the operating costs; also it supports system scalability in a responsible way, according to the demand of mobile users and smart metering.

The simulations shown above demonstrate the proper way to send information from smart meters; this transmission is associated to the coverage and traffic. Traffic limits the use of wireless networks by reducing the amount of information to be transmitted.

\section{Future WORK}

Proper planning of resources and networks to reuse the cellular network as a means of communication is vital to send information. Using various optimization algorithms, future work is expected to be centered on a study of the capacity of the channels to determine an optimal distribution of information and thus avoiding overcrowding the spectrum capacity and reducing noise in communications

\section{REFERENCES}

[1] R. F. Arritt and R. C. Dugan, "Distribution System Analysis and the Future Smart Grid," IEEE Trans. Ind. Appl., vol. 47, no. 6, pp. 2343-2350, Nov. 2011. DOI: 10.1109/REPCON.2011.5756725

[2] J. Kim, S. Cho, and H. Shin, "Advanced Power Distribution System Configuration for Smart Grid," IEEE Trans. Smart Grid, vol. 4, no. 1, pp. 353-358, 2013. DOI: $10.1109 /$ TSG.2012.2233771

[3] C. Selva, K. Srinivas, G. Ayyappan, and M. Venkatachala, "Advanced Metering Infrastructure for Smart Grid Applications," Recent Trends Inf. Technol. (ICRTIT), 2012 Int. Conf. IEEE, pp. 145-150, 2012. DOI: 10.1109/ICRTIT.2012.6206777 
[4] S. SAydjari and V. Varadharajan, "The Smarter Grid," Secur. Privacy, IEEE, vol. 8, no. 1, pp. $60-63,2010$. DOI: 10.1109/MSP.2010.52

[5] L. Chun-hao and N. Ansari, "CONSUMER: A Novel Hybrid Intrusion Detection System for Distribution Networks in Smart Grid," IEEE Trans. Emerg. Top. Comput., vol. 1, no. 1, pp. 33 - 44, 2013. DOI: 10.1109/ TETC.2013.2274043

[6] E. Inga and J. Inga, "Wireless Communications and Virtual Operator for Residential Electric Metering," 12th Lat. Am. Caribb. Conf. Eng. Technol., pp. 1-9, 2014.

[7] B. Karimi and V. Namboodiri, "On the Capacity of a Wireless Backhaul for the Distribution Level of the Smart Grid," Syst. Journal, IEEE, vol. 8, no. 2, pp. 521-532, 2014. DOI: 10.1109/JSYST.2013.2260701

[8] E. Inga, R. Hincapie, C. Suarez, and G. Arevalo, "Shortest path for optimal routing on Advanced Metering Infrastructure using cellular networks," in Communications and Computing (COLCOM), 2015 IEEE Colombian Conference on, 2015, pp. 1 - 6. DOI: 10.1109/ColComCon.2015.7152078

[9] Z. Jia, J. Chen, and Y. Liao, "State estimation in distribution system considering effects of AMI data," 2013 Proc. IEEE Southeastcon, pp. 1-6, Apr. 2013. DOI: 10.1109/ SECON.2013.6567406

[10] R. R. Mohassel, A. Fung, F. Mohammadi, and K. Raahemifar, "Application of Advanced Metering Infrastructure in Smart Grids," Control Autom. (MED), 2014 22nd Mediterr. Conf. IEEE, pp. 822 - 828, 2014. DOI: 10.1109/ MED.2014.696147

[11] C. Lo and N. Ansari, "The Progressive Smart Grid System from Both Power and Communications Aspects," Commun. Surv. Tutorials, IEEE, vol. 14, no. 3, pp. 799821, 2012. DOI: 10.1109/SURV.2011.072811.00089

[12] P. Kulkarni, S. Gormus, Z. Fan, and F. Ramos, "AMI Mesh Networks-A Practical Solution and Its Performance Evaluation," IEEE Trans. Smart Grid, vol. 3, no. 3, pp. 1469-1481, Sep. 2012. DOI: 10.1109/TSG.2012.2205023

[13] E. Hossain, Z. Han, and H. V. Poor, Smart Grid communication and networking, First edit. United Stated of America: Cambridge University Press, 2012, p. 481.

[14] H. Sui and W.-J. Lee, "An AMI based measurement and control system in smart distribution grid," 2011 IEEE Ind. Commer. Power Syst. Tech. Conf., pp. 1-5, May 2011. DOI: $10.1109 /$ ICPS.2011.5890876

[15] C. Andres and C. John, The Advanced Smart Grid Edge Power Driving Sustainability, First Edit. 2011, p. 268.

[16] M. R. Souryal and N. Golmie, "Analysis of advanced metering over a Wide Area Cellular Network," 2011 IEEE Int. Conf. Smart Grid Commun., pp. 102-107, Oct. 2011. DOI: 10.1109/SmartGridComm.2011.6102299

[17] B. Saint, "Rural Distribution System Planning using Smart Grid Technologies," Rural Electr. Power Conf. 2009. REPC '09. IEEE, no. 09, pp. 0-8, 2009. DOI: 10.1109/REPCON.2009.4919421

[18] S. Li, J. Huang, and S. Member, "Dynamic Profit Maximization of Cognitive Mobile Virtual Network Operator," IEEE Trans. Mob. Comput., vol. 13, no. 3, pp. 526-540, 2014. DOI: $10.1109 /$ TMC.2013.10

[19] M. Gupta, S. Gupta, and T. Thakur, "Design and impact of wireless AMI for distribution utilities," 2013 Annu. IEEE India Conf., pp. 1-6, Dec. 2013. DOI: 10.1109/INDCON.2013.6726103

[20] H. Li, S. Gong, L. Lai, Z. Han, S. Member, R. C. Qiu, and D. Yang, "Efficient and Secure Wireless Communications for Advanced Metering Infrastructure in Smart Grids," IEEE Trans. Smart Grid, vol. 3, no. 3, pp. 1540-1551, 2012. DOI: $10.1109 /$ TSG.2012.2203156
[21] A. Peralta-Sevilla, E. Inga, R. Cumbal, and R. Hincapie, "Optimum deployment of FiWi Networks using wireles sensors based on Universal Data Aggregation Points," in Communications and Computing (COLCOM), 2015 IEEE Colombian Conference on, 2015, pp. 1 - 6. DOI: 10.1109/ ColComCon.2015.7152079

[22] I. E. Reid and H. A. Stevens, Smart Meters and the Smart Grid: Privacy and Cybersecurity Considerations, First edit. Nova Science Publishers, Incorporated, 2012, p. 153.

[23] S. Cespedes, A. A. Cardenas, and T. Iwao, "Comparison of Data Forwarding Mechanisms for AMI networks," Innov. Smart Grid Technol. (ISGT), 2012 IEEE PES, pp. $1-8,2012$. DOI: 10.1109/ISGT.2012.6175683

[24] C. Scordino and G. Lipari, "A Resource Reservation Algorithm for Power-Aware Scheduling of Periodic and Aperiodic Real-Time Tasks," Comput. IEEE Trans., vol. 55, no. 12 , pp. 1509 - 1522, 2006. DOI: 10.1109/TC.2006.190

[25] L. Duan, J. Huang, and B. Shou, "Cognitive Mobile Virtual Network Operator: Investment and Pricing with Supply Uncertainty," INFOCOM, 2010 Proc. IEEE, pp. $1-9,2010$. DOI: $10.1109 /$ INFCOM.2010.5462146

[26] Z. Fan and R. Zhang, "Spectrum allocation and medium access in cognitive radio wireless networks," 2009 Eur. Wirel. Conf. IEEE, pp. 90-95, May 2009. DOI: 10.1109/ EW.2009.5357757

[27] L. Mastroeni and M. Naldi, "Spectrum reservation options for Mobile Virtual Network Operators," IEEE 6th EURO-NGI Conf. Next Gener. Internet, vol. 13, p. 15, Jun. 2010. DOI: 10.1109/NGI.2010.5534477

[28] E. Inga, G. Arévalo, and R. Hincapié, "Optimal Deployment of Cellular Networks for Advanced Measurement Infrastructure in Smart Grid," 2014 IEEE Colomb. Conf. Commun. Comput., no. 1The 2014 IEEE Colombian Conference on Communications and Computing, p. 6, 2014 DOI: 10.1109/ColComCon.2014.6860421

[29] A. D. Dabbagh, R. Ratasuk, and A. Ghosh, "On UMTS LTE Physical Uplink Shared and Control Channels," Veh. Technol. Conf. 2008. VTC 2008-Fall. IEEE 68th, pp. $1-8,2008$. DOI: 10.1109/VETECF.2008.323

[30] R. Agrawal, R. Berry, J. Huang, and V. Subramanian, "Optimal Scheduling for OFDMA Systems," Signals, Syst. Comput. 2006. ACSSC '06. Fortieth Asilomar Conf. IEEE, pp. 1347 - 1351, 2006. DOI: 10.1109/ ACSSC.2006.354976

[31] S. Sesia, I. Toufik, and M. Baker, LTE the UMTS Long Term Evolution from theory to practice, Second ed. Great Britain, 2011, p. 794.

[32] J. Markkula and J. Haapola, "Impact of smart grid traffic peak loads on shared LTE network performance," Commun. (ICC), 2013 IEEE Int. Conf., pp. 4046 - 4051, 2013. DOI: 10.1109/ICC.2013.6655193

[33] X. Qin and R. Berry, "Distributed Resource Allocation and Scheduling in OFDMA Wireless Networks," Sig. nals, Syst. Comput. 2006. ACSSC '06. Fortieth Asilomar Conf. IEEE, pp. 1942 - 1946, 2006. DOI: 10.1109/ ACSSC.2006.355102

[34] E. Ortega, D. Cazco, V. Luna, and J. Ortega, "Comunicaciones celulares para medición inteligente de energía eléctrica en sistemas de distribución," ingenius.ups.edu. ec, pp. 21-33, 2013

[35] I. Joe, J. Y. J. Zhang, and Fu-Quan, "Design and Implementation of AMI System Using Binary CDMA for Smart Grid," Intell. Syst. Des. Eng. Appl. (ISDEA), 2013 Third Int. Conf. IEEE, pp. 544 - 549, 2013. DOI: 10.1109/ ISDEA.2012.133

[36] K. S. Zigangirov, Theory Of Code Division Multiple Access Communication, First edit. Canada, 2004, p. 412. 